\title{
CORMAN: A Novel Cooperative Opportunistic Routing Scheme in Mobile Ad Hoc Networks
}

\author{
Zehua Wang, Student Member, IEEE, Yuanzhu Chen, Member, IEEE, Cheng Li, Senior Member, IEEE
}

\begin{abstract}
The link quality variation of wireless channels has been a challenging issue in data communications until recent explicit exploration in utilizing this characteristic. The same broadcast transmission may be perceived significantly differently, and usually independently, by receivers at different geographic locations. Furthermore, even the same stationary receiver may experience drastic link quality fluctuation over time. The combination of link-quality variation with the broadcasting nature of wireless channels has revealed a direction in the research of wireless networking, namely, cooperative communication. Research on cooperative communication started to attract interests in the community at the physical layer but more recently its importance and usability have also been realized at upper layers of the network protocol stack. In this article, we tackle the problem of opportunistic data transfer in mobile ad hoc networks. Our solution is called Cooperative Opportunistic Routing in Mobile Ad hoc Networks (CORMAN). It is a pure network layer scheme that can be built atop off-the-shelf wireless networking equipment. Nodes in the network use a lightweight proactive source routing protocol to determine a list of intermediate nodes that the data packets should follow en route to the destination. Here, when a data packet is broadcast by an upstream node and has happened to be received by a downstream node further along the route, it continues its way from there and thus will arrive at the destination node sooner. This is achieved through cooperative data communication at the link and network layers. This work is a powerful extension to the pioneering work of ExOR. We test CORMAN and compare it to AODV, and observe significant performance improvement in varying mobile settings.
\end{abstract}

Index Terms-Cooperative communication, opportunistic routing, opportunistic forwarding, mobile ad hoc networks, proactive source routing, local retransmission, forwarder list update

\section{INTRODUCTION}

A MOBILE ad hoc network is a wireless communication network, where nodes that are not within direct transmission range of each other will require other nodes to forward data. It can operate without existing infrastructure, supports mobile users, and falls under the general scope of multi-hop wireless networking. Such a networking paradigm originated from the needs in battlefield communications, emergence operations, search and rescue, and disaster relief operations. Later, it found civilian applications such as community networks. A great deal of research results have been published since its early days in the 1980's [1]. The most salient research challenges in this area include end-to-end data transfer, link access control, security, and providing support for real-time multimedia streaming.

Manuscript received 14 April 2011; revised 20 July 2011. This work was supported in part by the Natural Sciences and Engineering Research Council (NSERC) of Canada (Discovery Grants 293264-07 and 327667-2010, and Strategic Project Grant STPGP 397491-10).

The authors are with Memorial University of Newfoundland (e-mail: \{zehua.wang,yzchen,licheng\}@mun.ca).

Digital Object Identifier 10.1109/JSAC.2012.120207.
The network layer has received the most attention when working on mobile ad hoc networks. As a result, abundant routing protocols in such a network with differing objectives and for various specific needs have been proposed [2]. In fact, the two most important operations at the network layer, i.e., data forwarding and routing, are distinct concepts. Data forwarding regulates how packets are taken from one link and put on another. Routing determines which path a data packet should follow from the source node to the destination. The latter essentially provides the former with control input.

Although a large amount of efforts have been put in routing in ad hoc networks, in contrast, data forwarding follows pretty much the same paradigm as in IP forwarding in the Internet. IP forwarding was originally designed for multi-hop wired networks, where one packet transmission can only be received by nodes attached to the same cable. For the case of modern Ethernet, an IP packet is transmitted at one end of the Ethernet cable and received at the other. However, in wireless networks, when a packet is transmitted over a physical channel, it can be detected by all other nodes within the transmission range on that channel. For the most part of the research history, overhearing a packet not intended for the receiving node had been considered as completely negative, i.e., interference. Thus, the goal of research in wireless networking was to make wireless links as good as wired ones. Unfortunately, this ignores the inherent nature of broadcasting of wireless communication links. For mobile ad hoc networks to truly succeed beyond labs and testbeds, we must tame and utilize its broadcasting nature rather than fighting it. Cooperative communication is an effective approach to achieving such a goal.

Research on cooperative commutation at the link layer and above had been little until ExOR [3]. ExOR is a milestone piece of work in this area and it is an elegant way to utilize the broadcasting nature of wireless links to achieve cooperative communication at the link and network layers of static multihop wireless networks. Here, we further extend the scenarios that the idea behind ExOR can be used, dubbed as Cooperative Opportunistic Routing in Mobile Ad hoc Networks (CORMAN). We test CORMAN using the Nakagami fading model in ns-2 and compare it to the well-understood AODV in an array of mobile network scenarios. The performance improvement of CORMAN that we have observed is substantial. Contributions in our solution are highlighted as follows.

- We use a lightweight proactive source routing protocol so that each node has complete knowledge of how to route data to all other nodes in the network at any time. When a flow of data packets are forwarded towards their destination, the route information carried by them 
can be adjusted by intermediate forwarders. Furthermore, as these packets are forwarded along the new route, such updated information is propagated upstream rapidly without any additional overhead. As a result, all upstream nodes learn about the new route at a rate much faster than via periodic route exchanges.

- We take opportunistic data forwarding to another level by allowing nodes that are not listed as intermediate forwarders to retransmit data if they believe certain packets are missing.

The rest of this article is organized as follows. Section II reviews related work on utilizing the broadcasting nature of wireless links, with special focus on ExOR. Section III describes the basic idea of CORMAN. The details of CORMAN are presented in Sections IV and V. We use computer simulation to test the performance of CORMAN, and the settings and results of these experiments are in Section VI. In Section VII, we discuss some of our crucial design choices and their effects on the overall of CORMAN. Section VIII concludes the article with an outlook to future research.

\section{RELATED WORK}

The utilization of the broadcasting nature of wireless channels at the link layer and above has a relatively recent history compared to the efforts at the physical layer. Larsson [4] proposes an innovative handshake technique, called Selection Diversity Forwarding (SDF), to implement downstream forwarder selection in a multihop wireless network, where multiple paths are provided by the routing module. In this case, a sender in the network can dynamically choose from a set of usable downstream neighbors that present high transient link quality. For the sender to make the decision, the IEEE 802.11 Distributed Coordination Function (DCF)-based DATA/ACK handshake is enhanced. Such a handshake is the first opportunistic utilization of link quality variation in multihop wireless networks at the link and network layers. The coordination in SDF is somewhat expensive and its overhead needs to be significantly reduced for it to be more practical.

ExOR [3] is an answer to that. It is an explorative crosslayer opportunistic data forwarding technique in multi-hop wireless networks by Biswas and Morris. It fuses the MAC (Medium Access Control) and network layers so that the MAC layer can determine the actual next-hop forwarder after transmission depending on the transient channel conditions at all eligible downstream nodes. Nodes are enabled to overhear all packets transmitted in the channel, whether intended for it or not. A multitude of forwarders can potentially forward a packet as long as it is included on the forwarder list carried by the packet. Thus, if a packet is heard by a listed forwarder closer to the destination with a good reception condition, this long-haul transmission should be utilized. Otherwise, shorter and thus more robust transmissions can always be used to guarantee reliable progress. The challenge is to ensure that exactly one of the listed forwarders should relay the packet that is likely to be the closest to the destination at the same time. This is addressed by prioritized scheduling among the listed forwarders according to their priority indicated in the forwarder list.
We adopt the following terms defined in ExOR to describe similar concepts in CORMAN:

- Batch size - number of packets in a batch. It has the same value for all packets in a given batch.

- Forwarder list size - number of forwarders on the forwarder list. It has the same value for all packets in a given batch.

- Packet number - index of the packet in the batch.

- Forwarder number - index of the forwarder on the forwarder list. It indicates which node on the list has just transmitted the data packet.

- Batch map - an array whose size equals the batch size. Each element of the map is indexed by the packet number, and its value is the forwarder number of the highestpriority forwarder that this packet has reached.

- Fragment - a subset of packets in the current batch which are sent together from a given forwarder.

In ExOR, data packets are prepended with an ExOR header, which includes some of the above information. They are further prepended with an 802.11 DATA frame header before being broadcasted. For more details of the packet formats, the readers are referred to [3].

The idea of ExOR has since inspired a number of interesting extensions. Here, we just name a few of these without trying to be complete. MORE [5] enhances ExOR to further increase the spatial reuse in a single flow from source to destination via intra-flow network coding [6]. Leontiadis and Mascolo [7] and Yang et al. [8] propose using position information for the routing module to support mobile multi-hop wireless networks. Therein, it is assumed that every node is aware of the positions of all other nodes in the network. This is possible if some sort of universal positioning service and out-of-band dissemination of such information in the network are available.

To generalize the idea behind ExOR to more different types of networks, such as mobile wireless networks, lightweight routing algorithms with proactive source routing capabilities are preferred. Path Finding Algorithm (PFA) [9] was developed based on the distance vector algorithm, which incorporates the predecessor of a destination in a routing update. Hence, the entire path to a destination can be reconstructed by the source node. On the other hand, the Link Vector (LV) algorithm [10] reduces the overhead of link-state algorithms to a great deal by only including links to be used in data forwarding in routing updates. The extreme case of LV where only one link is included per destination coincides with PFA. While the two algorithms above were initially proposed to address the routing scalability issue in the Internet, the Wireless Routing Protocol (WRP) [11] attempts to apply the idea of PFA to mobile wireless networks. The update messages in WRP are event-driven and it requires reliable link-layer broadcast, which incurs a significant amount of overhead.

\section{OVERVIEW OF CORMAN}

CORMAN is a network layer solution to the opportunistic data transfer in mobile ad hoc networks. Its node coordination mechanism is largely inline with that of ExOR and it is an extension to ExOR in order to accommodate node mobility. Here, we first highlight our objectives and challenges in order 
to achieve them. Later in this section, we provide a general description of CORMAN. The details of CORMAN will be presented in Sections IV and V.

\section{A. Objectives and challenges}

CORMAN has two objectives. 1) It broadens the applicability of ExOR to mobile multi-hop wireless networks without relying on external information sources, such as node positions. 2) It incurs a smaller overhead than ExOR by including shorter forwarder lists in data packets.

The following challenges are thus immanent.

1) Overhead in route calculation - CORMAN relies on the assumption that every source node has complete knowledge of how to forward data packets to any node in the network at any time. This calls for a proactive source routing protocol. Link state routing, such as OLSR, would meet our needs but it is fairly expensive in terms of communication costs. Therefore, we need a lightweight solution to reduce the overhead in route calculation.

2) Forwarder list adaptation - When the forward list is constructed and installed in a data packet, the source node has updated knowledge of the network structure within its proximity but its knowledge about further areas of the network can be obsolete due to node mobility. This error becomes worse as the data packet is forwarded towards the destination node. To address this issue, intermediate nodes should have the ability to update the forwarder list adaptively with their new knowledge when forwarding data packets.

3) Robustness against link quality variation - When used in a dynamic environment, a mobile ad hoc network must inevitably face the drastic link quality fluctuation. A short forwarder list carried by data packets implies that they tend to take long and possibly weak links. For opportunistic data transfer, this could be problematic since the list may not contain enough redundancy in selecting intermediate nodes. This should be overcome with little additional overhead.

\section{B. CORMAN fundamentals}

CORMAN forwards data in a similar batch-operated fashion as ExOR. A flow of data packets are divided into batches. All packets in the same batch carry the same forwarder list when they leave the source node. To support CORMAN, we have an underlying routing protocol, Proactive Source Routing (PSR), which provides each node with the complete routing information to all other nodes in the network. Thus, the forwarder list contains the identities of the nodes on the path from the source node to the destination. As packets progress in the network, the nodes listed as forwarders can modify the forwarder list if any topology change has been observed in the network. This is referred to as large-scale live update in our work. In addition, we also allow some other nodes that are not listed as forwarders to retransmit data if this turns out to be helpful, referred to as small-scale retransmission. Note that CORMAN is a completely network layer solution and can be built upon off-the-shelf IEEE 802.11 networking commodities without modification.

Therefore, the design of CORMAN has the following three modules. Each module answers to one of the challenges stated previously.

1) Proactive source routing - PSR runs in the background so that nodes periodically exchange network structure information. It converges after the number of iterations equal to the network diameter. At this point, each node has a spanning tree of the network indicating the shortest paths to all other nodes. PSR is inspired by path finding [9], [11] and link-vector [10] algorithms but is lighter weight. Technically, PSR can be used without CORMAN to support conventional IP forwarding.

2) Large-scale live update - When data packets are received by and stored at a forwarding node, the node may have a different view of how to forward them to the destination from the forwarder list carried by the packets. Since this node is closer to the destination than the source node, such discrepancy usually means that the forwarding node has more updated routing information. In this case, the forwarding node updates the part of the forwarder list in the packets from this point on towards the destination according to its own knowledge. When the packets with this updated forwarder list are broadcast by the forwarder, the update about the network topology change propagates back to its upstream neighbor. The neighbor incorporates the change to the packets in its cache. When these cached packets are broadcast later, the update is further propagated towards the source node. Such an update procedure is significantly faster than the rate at which a proactive routing protocol disseminates routing information.

3) Small-scale retransmission - A short forwarder list forces packets to be forwarded over long and possibly weak links. To increase the reliability of data forwarding between two listed forwarders, CORMAN allows nodes that are not on the forwarder list but are situated between these two listed forwarders to retransmit data packets if the downstream forwarder has not received these packets successfully. Since there may be multiple such nodes between a given pair of listed forwarders, CORMAN coordinates retransmission attempts among them extremely efficiently.

The details of PSR and its improvements are in separate work of ours [12], [13]. The second and third modules are described in subsequent sections.

\section{LARGE SCALE LIVE UPDATE}

CORMAN generalizes the opportunistic data forwarding in ExOR to suit mobile wireless networks. That is, when a batch of packets are forwarded along the route towards the destination node, if an intermediate node is aware of a new route to the destination, it is able to use this new route to forward the packets that it has already received. There are a few implications of this. First, this new route will also be used to forward the subsequent packets of the same batch. Second, when packets are forwarded along the new route, such 


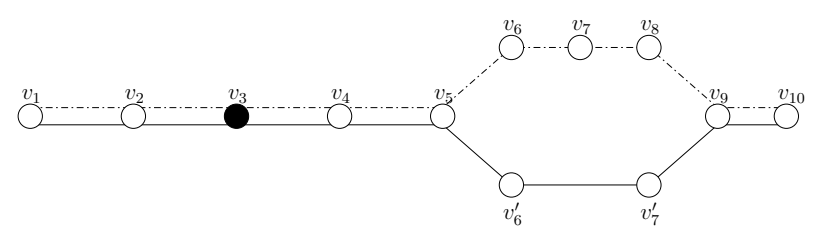

Fig. 1. Route update

an updated forwarder list replaces the old list in the packets. By overhearing the forwarding of these packets, the upstream nodes can be notified of the new route and this information can propagate back to the source node quickly. Details of data forwarding and list update are described in this section with the help of Figure 1. In the figure, the source node $v_{1}$ has a flow of data packets for destination node $v_{10}$. According to its own routing module, $v_{1}$ decides that the best route to $v_{10}$ is $v_{1} v_{2} v_{3} v_{4} v_{5} v_{6} v_{7} v_{8} v_{9} v_{10}$; hence the forwarder list.

At a given point of time during the data transfer of a batch, there is a node on the forwarder list that has the highest priority and has received any packet of the batch. We call such a node the frontier of the batch. At the beginning, the frontier is the source node. When the destination has received at least one packet of a batch, it has become the frontier of the batch. Recall that a fragment (Section II) is a subset of packets in the current batch which are transmitted in tandem by a given forwarder. Here, the frontier has cached its first fragment of packets. Suppose at this point, the frontier in Figure 1 is $v_{3}$. When it is about to forward this fragment, if its routing module indicates there is a new route to the destination, e.g., $v_{3} v_{4} v_{5} v_{6}^{\prime} v_{7}^{\prime} v_{9} v_{10}$, it replaces the segment of the original forwarder list from itself to the destination (i.e., $v_{3} v_{4} v_{5} v_{6} v_{7} v_{8} v_{9} v_{10}$ ) with this new route. That is, the forwarder list carried by these data packets are now $v_{1} v_{2} v_{3} v_{4} v_{5} v_{6}^{\prime} v_{7}^{\prime} v_{9} v_{10}$. When the packets of the fragment are forwarded, they will be following the new route. In addition, upstream nodes can overhear these packets, and thus their new forwarder list. These nodes can update their own routing information and will incorporate such information when forwarding their fragments. This backtrack continues until the source is aware of the latest route information. We would like to bring up the following notes to the readers' attention.

1) When the network diameter is large and nodes are moving fast, the routing information can be obsolete by the time it has propagated to a remote node. That is, a node's knowledge about the network topology becomes less accurate when the destination node is located farther away. Thus, the forwarder list composed by the source node needs to be adjusted as packets are forwarded towards the destination, where intermediate nodes closer to the destination could have better routing information. This is achieved effectively by allowing the frontier node to modify the forwarder list carried by the fragment of packets. As a result, CORMAN has a fairly good tolerance of route inaccuracy for any source node to start with.

2) When a frontier node updates an forwarder list, only the segment of the list between the frontier and destination is replaced while the rest of the list (i.e., nodes that the

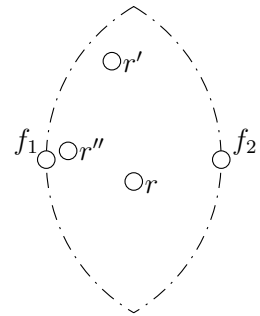

Fig. 2. Retransmission region

fragment have gone through) are intact. The reason for this design decision is that these upstream nodes should not be disturbed by the new route so that the scheduling coordination among them is consistent.

3) We only allow a frontier node to update a packet's forwarder list according to its routing module. A node that is no longer a frontier should only incorporate the forwarder list that it overhears from downstream nodes. The purpose is to avoid unnecessary updates of route information as the time needed to transfer a batch of data packets is very short. During this time, usually little has changed about the network topology and nodes may not even have exchanged the routing information for the next periodic interval.

4) Consider a particular intermediate node on the forwarder list. As the frontier moves from the source to the destination, the forwarder list may be refreshed multiple times by different frontiers. Thus, this node may experience one update about its route to the destination every time a frontier decides to modify the list.

All of the above is achieved rapidly and with no extra communication overhead compared to ExOR.

\section{SMALL SCALE RETRANSMiSSION}

To enhance the reliability of packet transmission between two consecutive listed forwarders, CORMAN employs the mechanism of small-scale retransmission. It operates at the time granularity of a fragment and space granularity of a single link. Consider a given batch of packet transfer and suppose that two consecutive forwarders on this batch's list are $f_{1}$ and $f_{2}$, in that order, as in Figure 2, and that a node $r$ is located somewhere between $f_{1}$ and $f_{2}$. After $f_{2}$ has transmitted its fragment of packets, by comparing the packets transmitted by $f_{1}$ to those by $f_{2}$, node $r$ knows which packets $f_{2}$ has missed. It is now eligible to retransmit these packets that are believed missing. If there are more than one such $r$ in that area, such as $r^{\prime}$ and $r^{\prime \prime}$, CORMAN guarantees at most one such node should retransmit. For $r$ to be a retransmitter in this case, it must satisfy the following conditions:

- First, node $r$ should be a neighbor of both $f_{1}$ and $f_{2}$. Node $r$ can learn this by looking up its neighbor list.

- Second, the separation distance between $f_{1}$ and $f_{2}$, denoted $d\left(f_{1}, f_{2}\right)$, should be greater than $d\left(f_{1}, r\right)$ and $d\left(r, f_{2}\right)$. That is, it must be within the region in Figure 2 enclosed by the dashed lines. The node separation distance can be estimated using the RSSI (Received Signal Strength Indicator) recorded when packets are received. 
- Third, node $r$ uses a scoring function $\mathbb{F}\left(d\left(f_{1}, r\right), d\left(r, f_{2}\right)\right)$ in the positive real domain to measure how "suitable" it is as a retransmitter for $f_{1}$ and $f_{2}$. F can be any function that favors a node close to the midpoint of the line segment between $f_{1}$ and $f_{2}$ but not too close to either one of them. In addition, node $r$ is also able to calculate this suitability value for all other nodes satisfying the first two conditions. This is possible if node $r$ is aware of the RSSI measurements of all links incident on a node in $N(r)$. This can be realized simply by every node broadcasting the RSSI of all incident links periodically. This last condition ensures that node $r$ should have the best suitability score among those satisfying the first two conditions.

Suppose $r$ decides that it is indeed the retransmitter between $f_{1}$ and $f_{2}$. It should retransmit the packets that $f_{2}$ or any higher-priority node has missed. For us to keep track of the progression of data packets in the batch, each element of the batch map can have a value $p(0 \leq p \leq 2(l-1))$, where $l$ is the number of nodes in the forwarder list. We use the parity of $p$ to distinguish if the corresponding node is a listed forwarder or not. That is, when it is even, $p / 2$ is the offset of the node in the list that the corresponding packet has reached. When $p$ is odd, the packet has reached the retransmitter between the $(p-1) / 2$-th and $(p+1) / 2$-th forwarders. Once node $r$ has learned what packets should be retransmitted and what batch map they should carry, it retransmits them as if it were a listed forwarder.

\section{Performance eVAluation}

In this section, we study the performance of CORMAN by running computer simulation using Network Simulator ns-2 (version 2.34). We compare it against AODV with varying network densities and node mobility rates. The performance improvement and explanations of these results are explained in the rest of this section.

\section{A. Experiment settings}

The channel propagation model used in ns-2 had been predominantly the Two-Ray Ground Reflection model early on. However, this model is realized to be a simplified a path loss model without considering fading. In our work, we choose the Nakagami propagation model to test CORMAN in a more realistic fading environment. In ns-2, when a node has received a packet, it first calculates the received power using path loss based on the Frii Free-Space model. This value is compensated with Nakagami's fluctuation before further processing. We configure the nominal data rate at the 802.11 links to $1 \mathrm{Mbps}$,

We compare the performance of CORMAN with that of AODV [14]. We select AODV as the baseline because AODV is a widely adopted routing protocol in MANETs, and its behavior both in ns-2 and real network operations is well understood by the research community.

In modeling node motion, we adopt the random waypoint model to generate the simulation scenarios. In this model, each node moves towards a series of target positions. The rate of velocity for each move is uniformly selected from $\left[0, v_{\max }\right]$. Once it has reached a target position, it may pause for a specific amount of time before moving towards the next position. In our tests, we have two series of mobile scenarios using this mobility model. The first series of scenarios have a fixed $v_{\max }$, a constant number of nodes, but varying network dimensions. The second series have the same network size and dimension but varying $v_{\max }$.

We inject CBR (constant bit rate) data flows in the network, which are carried by UDP. Specifically, a source node generates 50 packets every second, each has a payload of 1000 bytes. This translates to a traffic rate of $400 \mathrm{kbps}$ injected by a node. When comparing CORMAN to AODV, we record the packet delivery ratio (PDR), i.e. the fraction of packets received by the destination out of all the packets injected, and end-to-end delay average and variance. We observe that CORMAN outperforms AODV in terms of all of these metrics.

\section{B. Performance versus network dimension}

We first compare the performance of CORMAN and AODV with different network dimensions. Specifically, we have network tomographies of $l \times l\left(\mathrm{~m}^{2}\right)$, where $l=$ $250,300,350, \ldots, 1000$. We deploy 50 nodes in each of these network dimensions to test the protocols with differing node densities. These nodes move following the random waypoint model with $v_{\max }=10 \mathrm{~m} / \mathrm{s}$. For each dimension scenario, we test CORMAN and AODV's capabilities in transporting CBR data flows between a randomly selected source-destination pair. We repeat this process 5 times for a given scenario. We measure the PDR, end-to-end delay, and delay jitter for both protocols and average them over the 5 repetitions of each scenario, as plotted in Figures 3, 4, and 5, respectively.

We observe that CORMAN has a PDR (Figure 3 ) of about 95\% for dense networks (i.e., $250 \leq l \leq 500 \mathrm{~m}$ ). As the node density decreases, this rate gradually goes down to about 60\%. In contrast, AODV's PDR ranges between $60 \%$ and $80 \%$ for dense networks and quickly drops to around $20 \%$ for sparse networks. (We use a red plotting series to indicate the relative performance of CORMAN over PDR in all of our figures.) There are two reasons for the PDR penalty for AODV to operate in sparse networks. First, data packets are forwarded using traditional IP forwarding in AODV. When channel quality varies (as emulated by the Nakagami model), a packet may be lost at the link layer. After a few failed retransmits, it will be dropped by the network layer. CORMAN, however, is designed to utilize such link effects so that at least one downstream node would be available despite the link variation. CORMAN facilitates opportunistic data forwarding using the link quality diversity at different receivers and allows them to cooperate with each other with a minimal overhead. Consequently, CORMAN has a strong resilience to link quality fluctuation and node mobility. Second, the route search of AODV does not function well with unreliable links. Recall that, in AODV, when a node finds that it does not have a next hop available for a given data packet, it broadcasts a RREQ (route request) to find one. Both the destination and any intermediate node that has a valid cached route can reply with a RREP. When links were perfectly symmetric, the RREP packet would take the inverse path leading to the initiating node of the route search. However, when links suffer from 


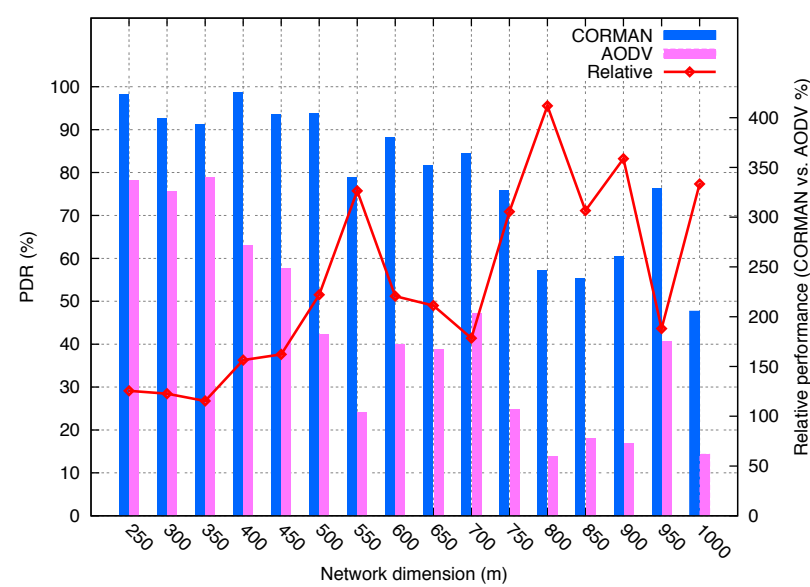

Fig. 3. PDR vs. network dimension

fading, the RREP packets may not be able to propagate back to the initiator because of transient low link quality in the reverse direction. As a result, it takes AODV much longer to obtain stable routes. In fact, this performance loss has been observed in earlier studies of AODV when large numbers of unidirectional links are present in the network [15]. In our ns-2 simulation using the Nakagami propagation model, the independent link quality fluctuation in both directions essentially produces temporary unidirectional links. This negative effect of fading links on AODV can be mediated to a degree when the node density increases. Therefore, in our next set of tests (Section VI-C), all simulation scenarios have a fairly high node density for AODV to function reasonably well.

We are also interested in the end-to-end delay and its variance of CORMAN and AODV. Figure 4 presents the end-to-end delay of these protocols in different dimension scenarios. We see that, when the node density is higher (i.e., $250 \leq l \leq 500 \mathrm{~m}$ ), CORMAN has a shorter delay than AODV. For sparser scenarios (i.e., $550 \leq l \leq 1000 \mathrm{~m}$ ), CORMAN's delay is slightly longer than AODV but comparable. In CORMAN's implementation, a node determines that it can no longer contribute to a batch's progression if it has not seen an update of the map after 10 retransmits of its fragment. This is similar to 10 retries at the link layer in IP style forwarding. (The default retry limit in 802.11 is 7 .) Thus, the not-so-short end-to-end delay of CORMAN is caused by the larger number of data retransmits. This is a relatively small cost to pay for a significantly higher PDR (Figure 3). The delay jitter (standard deviation) measured for CORMAN and AODV has similar relative performance for these scenarios as in Figure 5. This is also because of the larger retry limit of CORMAN (10 times) compared to AODV over 802.11 ( 7 times).

\section{Performance versus velocity}

We also study CORMAN's performance and compare it to AODV in different rates of node velocity. We conduct another set of tests in a network of 100 nodes deployed in a $300 \times 300\left(\mathrm{~m}^{2}\right)$ space with a varying $v_{\max }$, where $v_{\max }=0,2,4,6, \ldots, 20(\mathrm{~m} / \mathrm{s})$. For each velocity scenario, we test CORMAN and AODV's performance in transporting CBR data flows again between a randomly selected sourcedestination pair. We repeat this process 5 times for a given scenario. We collect the PDR, end-to-end delay, and delay

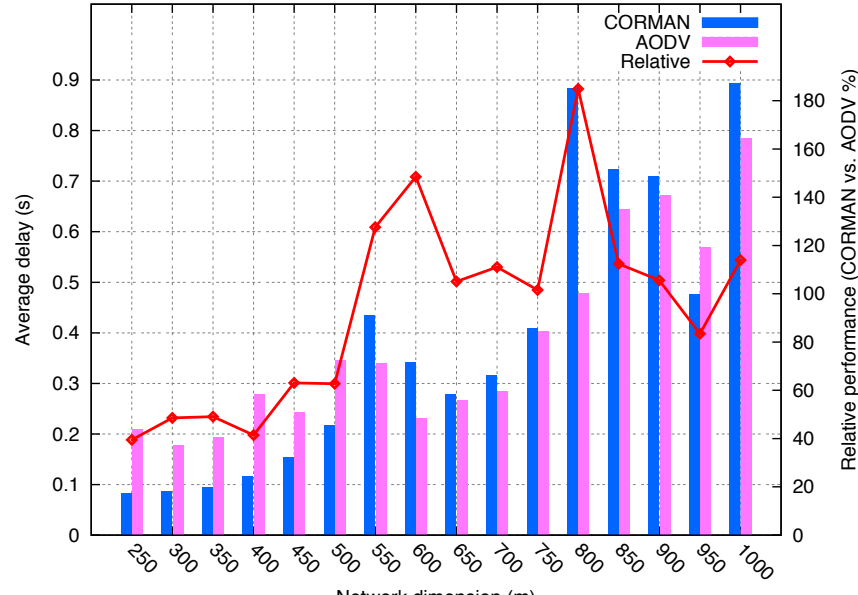

Fig. 4. Packet delay vs. network dimension

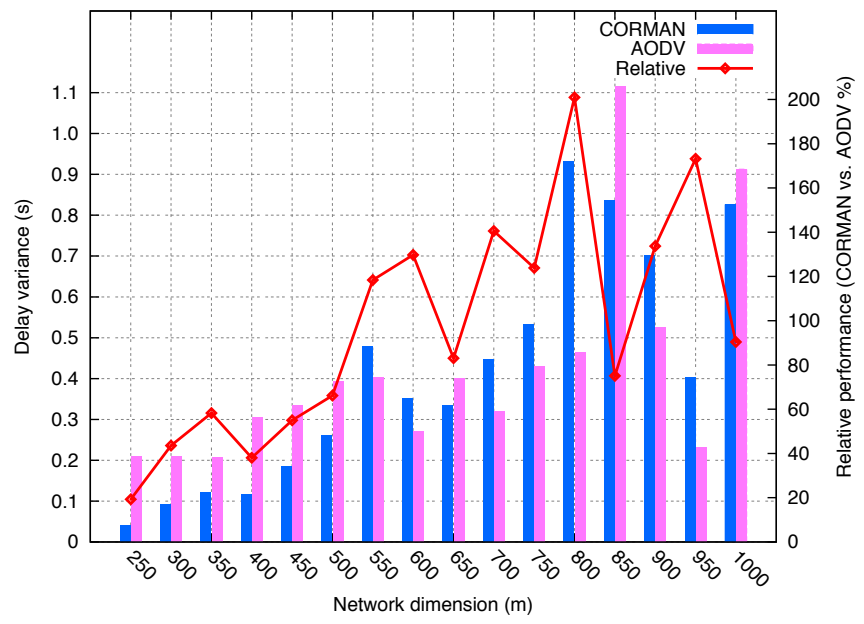

Fig. 5. Delay jitter vs. network dimension

jitter for both protocols averaged over each scenario, and plot them in Figures 6, 7, and 8, respectively. Note that these are fairly dense networks so that AODV has a reasonably high PDR. Since the network diameter is rather small in this case, the measurements are fairly consistent across these different velocity scenarios.

From Figure 6, we observe that CORMAN's PDR is constantly around 95\% while that of AODV varies between 57\% and $82 \%$. With this very high network density, AODV's route search succeeds in majority of the cases. Yet, it still does not have the same level of robustness against link quality changes as CORMAN. Compared to AODV, CORMAN has only a fraction of the end-to-end delay and variance (Figures 7 and 8) for two reasons. First, the opportunistic data forwarding scheme in CORMAN allows some packets to reach the destination in fewer hops than AODV. Second, the proactive routing (PSR) in CORMAN maintains full-on route information, whereas AODV still has to search for them if a route is broken. Although route search in AODV usually succeeds in dense networks with fluctuating link quality, the delay introduced by this process is inevitable.

Based on these observations, CORMAN can maintain its performance despite high rate of node velocity with realistic channels emulated by the Nakagami model. Hence, it is very suitable for a number of mobile ad hoc network applications, e.g., Vehicular Ad-hoc Network (VANET). 


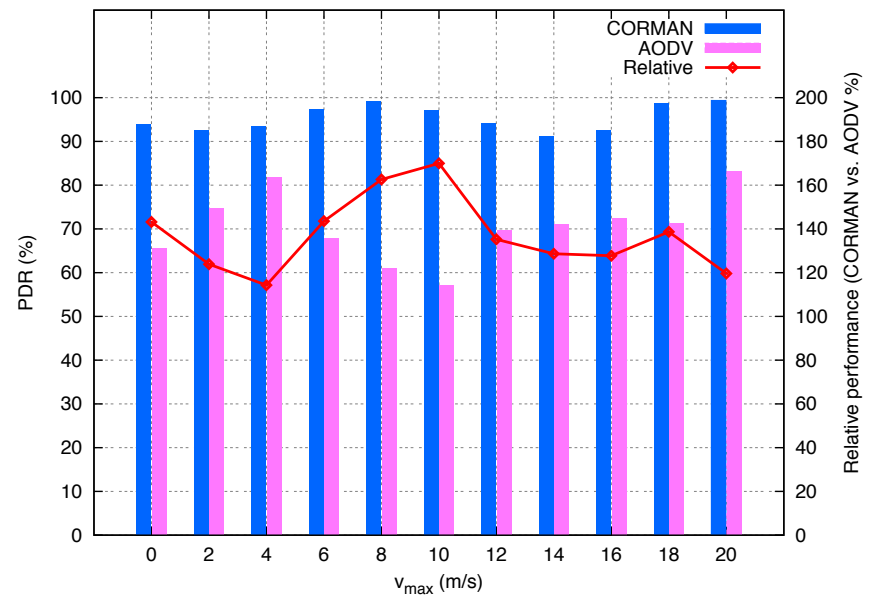

Fig. 6. PDR vs. node velocity

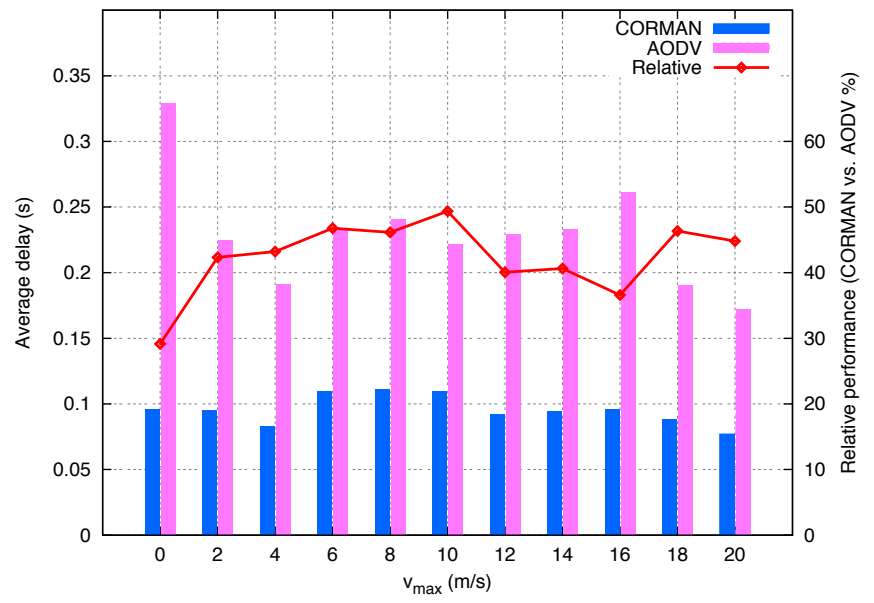

Fig. 7. Packet delay vs. node velocity

\section{DISCUSSION}

We would like to direct the readers' attention to the following discussion before concluding our article.

- The primary routing metric in PSR is hop count, with ETX to break ties. We decided to take this different approach from ExOR because we would like to utilize long links opportunistically as much as possible. Besides faster progression of packets from the source to the destination, the shorter forwarder lists also incur a smaller communication overhead in CORMAN in two aspects. First, the CORMAN protocol header is shorter compared to that of ExOR. Second, a compact forwarder list simplifies and expedites the coordination of downstream nodes in that a node in CORMAN does not need to wait for a larger number of higher-priority nodes before it can go forth to transmit the data. This inevitably pushes CORMAN to include forwarders linked by weak channels in data packets. The unreliability of these links is overcome by allowing nodes between consecutive forwarders to retransmit missing data packets.

- CORMAN is a network layer solution and its coupling with the link layer is minimal. Since the only 802.11 link layer service used by CORMAN is the unreliable broadcast of DATA frames, it can be built using off-theshelf equipment. As with ExOR, CORMAN implements part of the functionality which would otherwise have ex-

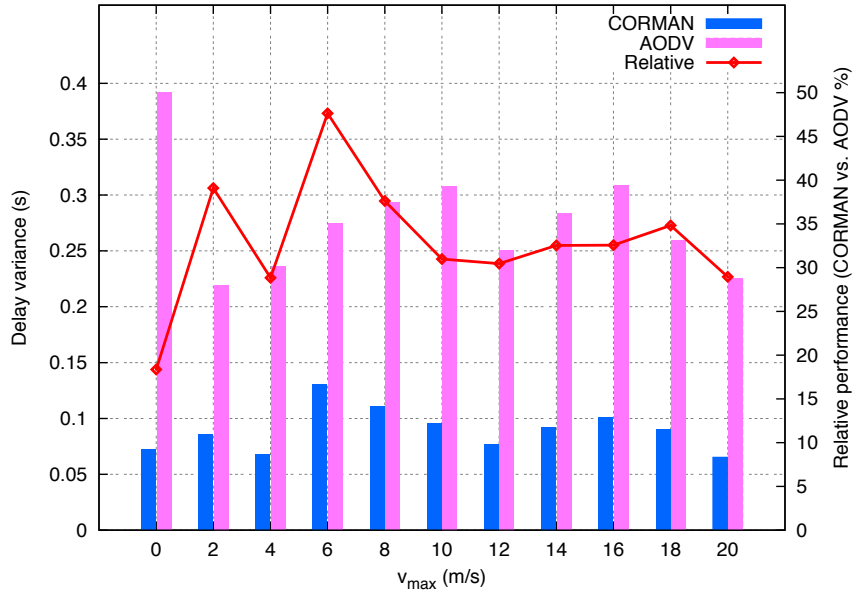

Fig. 8. Delay jitter vs. node velocity

isted at the link layer. For example, the acknowledgement of receiving packets is conveyed implicitly by the batch maps carried in the packets forwarded by downstream nodes.

- Our framework is completely compatible with the IEEE 802.11 standard family and can co-exist with conventional 802.11 traffic in the same network. After all, CORMAN is encapsulated as generic payload in 802.11 broadcast DATA frames.

- CORMAN is designed to support multiple simultaneous data flows. When a node overhears a packet from a nearby node, it records the time that this happens to estimate how long it will take that node to complete transmitting all packets in the fragment. The overhearing node uses this estimate to decide when it should start transmitting its own fragment. Such an estimation incorporates the backoff time of the DATA frame in order to reflect the traffic load in the network. As a result, if multiple flows are competing for the network resources, CORMAN allows them to share the network in an orderly fashion.

\section{CONCLUSION AND FUTURE WORK}

In this article, we have proposed CORMAN as an opportunistic routing scheme for mobile ad hoc networks. CORMAN is composed of three components. 1) PSR - a proactive source routing protocol, 2) large-scale live update of forwarder list, and 3) small-scale retransmission of missing packets. All of these explicitly utilize the broadcasting nature of wireless channels and are achieved via efficient cooperation among participating nodes in the network. Essentially, when packets of the same flow are forwarded, they can take different paths to the destination. For example, in Figure 9, the route between nodes $X$ and $Y$ as determined by the routing module is indicated by the yellow band. The solid circles represent the listed forwarders and the hollow ones are the small-scale retransmitters. In actual operations of CORMAN, packets $p_{1}$, $p_{2}$, and $p_{3}$ can take separate routes around this band depending on the transient link quality in the network. Such a decision is made on a per-hop and per-packet basis. Through computer simulation, CORMAN is shown to have superior performance measured in PDR, delay, and delay jitter. Research based on 


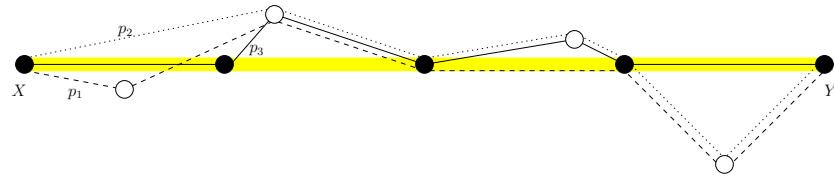

Fig. 9. Packet trajectories

CORMAN can be extended in the following interesting ways.

1) It would be informative to further test CORMAN. For example, we can compare CORMAN to ExOR and IP forwarding in static multi-hop networks with varying link quality to study their relative capabilities in data transfer. We will also test these data transfer techniques with multiple simultaneous flows are present to study how well they share the network resources. Through these tests, we will be able further optimize some parameters of CORMAN, such as the retry limit.

2) The coordination among multiple qualified small-scale retransmitters can be achieved with better measures than RSSI. In particular, if the "suitability" score is based on transient link quality rather than historical information, we will be able better utilize the receiver diversity. Apparently, this would require non-trivial coordination among these retransmitters, which could be challenging especially when we aim for zero extra overhead.

3) Nodes running CORMAN forward data packets in fragments. When the source and destination nodes are separated by many hops, it should allow nodes at different segment of the route to operate simultaneously. That is, a pipeline of data transportation could be achieved by better spatial channel reuse. The design of CORMAN can be further improved to address this explicitly. This may involve timing node backoff more precisely and tightly, or even devising a completely different coordination scheme.

The potential of cooperative communication in multi-hop wireless networks is yet to be unleashed at higher layers, and CORMAN is only an example.

\section{REFERENCES}

[1] I. Chlamtac, M. Conti, and J.-N. Liu, "Mobile Ad hoc Networking: Imperatives and Challenges," Ad Hoc Networks, vol. 1, no. 1, pp. 1364, July 2003.

[2] R. Rajaraman, "Topology Control and Routing in Ad hoc Networks: A Survey," SIGACT News, vol. 33, pp. 60-73, June 2002.

[3] S. Biswas and R. Morris, "ExOR: Opportunistic Multi-Hop Routing for Wireless Networks," in Proc. ACM Conference of the Special Interest Group on Data Communication (SIGCOMM), Philadelphia, PA, USA, August 2005, pp. 133-144.

[4] P. Larsson, "Selection Diversity Forwarding in a Multihop Packet Radio Network With Fading Channel and Capture," ACM Mobile Computing and Communications Review, vol. 5, no. 4, pp. 47-54, October 2001.

[5] S. Chachulski, M. Jennings, S. Katti, and D. Katabi, "Trading Structure for Randomness in Wireless Opportunistic Routing," in Proc. ACM Conference of the Special Interest Group on Data Communication (SIGCOMM), Kyoto, Japan, August 2007, pp. 169-180.

[6] C. Fragouli, J.-Y. L. Boudec, and J. Widmer, "Network Coding: an Instant Primer," SIGCOMM Computer Communication Review, vol. 36, pp. 63-68, January 2006.

[7] I. Leontiadis and C. Mascolo, "GeOpps: Geographical Opportunistic Routing for Vehicular Networks," in Proc. IEEE International Symposium on a World of Wireless Mobile and Multimedia Networks (WoWMoM), Helsinki, Finland, June 2007, pp. 1-6.
[8] S. Yang, F. Zhong, C. K. Yeo, B. S. Lee, and J. Boleng, "Position Based Opportunistic Routing for Robust Data Delivery in MANETs," in Proc. 2009 IEEE Conference on Global Telecommunications (GLOBECOM), Honolulu, Hawaii, USA, December 2009, pp. 1325-1330.

[9] S. Murthy, "Routing in Packet-Switched Networks Using Path-Finding Algorithms," Ph.D. dissertation, University of California - Santa Cruz, 1156 High Street, Santa Cruz, CA 95064, United States, 1996.

[10] J. Behrens and J. J. Garcia-Luna-Aceves, "Distributed, Scalable Routing based on Link-State Vectors," in Proc. ACM SIGCOMM, 1994, pp. 136147.

[11] S. Murthy and J. J. Garcia-Luna-Aceves, "An Efficient Routing Protocol for Wireless Networks," Mobile Networks and Applications, vol. 1, no. 2, pp. 183-197, October 1996.

[12] Z. Wang, C. Li, and Y. Chen, "PSR: Proactive Source Routing in Mobile Ad Hoc Networks," in Proc. 2011 IEEE Conference on Global Telecommunications (GLOBECOM), Houston, TX USA, December 2011.

[13] Z. Wang, Y. Chen, and C. Li, "A New Loop-Free Proactive Source Routing Scheme for Opportunistic Data Forwarding in Wireless Networks," IEEE Communications Letters, to appear.

[14] C. E. Perkins and E. M. Royer, "Ad hoc On-Demand Distance Vector (AODV) Routing," RFC 3561, July 2003. [Online]. Available: http://www.ietf.org/rfc/rfc3561.txt

[15] M. K. Marina and S. R. Das, "Routing Performance in the Presence of Unidirectional Links in Multihop Wireless Networks," in The Third ACM International Symposium on Mobile Ad Hoc Networking and Computing (MobiHoc'02), Lausanne, Switzerland, June 2002, pp. 12-23.

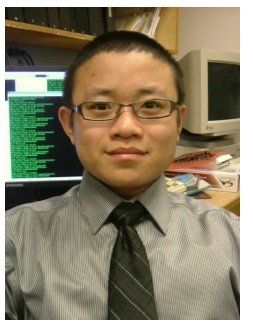

Zehua Wang is an M.Eng. student at Memorial University of Newfoundland, St. John's, Canada. He received his B.Eng. from Wuhan University, with Outstanding Thesis and Outstanding Graduand Awards, in 2009. He has served as the technical program committee (TPC) member for the IEEE ICC'12, IEEE GLOBECOM' 12 and IEEE WiMob'11. His research interests include wireless ad hoc networking, mobile and distributed computing, and generic data networks.

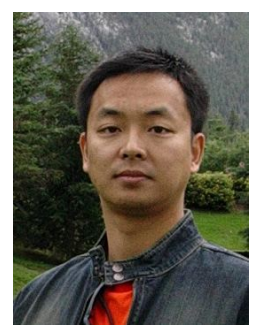

Yuanzhu Chen is an Associate Professor in the Department of Computer Science at Memorial University of Newfoundland, St. Johns, Newfoundland. He received his Ph.D. from Simon Fraser University in 2004 and B.Sc. from Peking University in 1999. Between 2004 and 2005 he was a post-doctoral researcher at Simon Fraser University. His research interests include computer networking, graph theory, Web information retrieval, evolutionary computation.

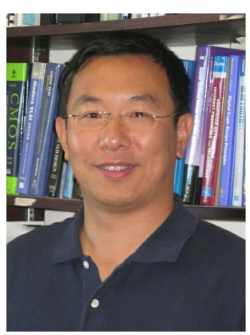

Cheng $\mathbf{L i}$ received the B.Eng. and M.Eng. degrees from Harbin Institute of Technology, Harbin, P. R. China, in 1992 and 1995, respectively, and the Ph.D. degree in Electrical and Computer Engineering from Memorial University, St. John's, Canada, in 2004.

$\mathrm{He}$ is currently an Associate Professor at the Faculty of Engineering and Applied Science of Memorial University, St. John's, Canada. His research interests include mobile ad hoc and wireless sensor networks, wireless communications and mobile computing, switching and routing, and broadband communication networks. He is an editorial board member of Wiley Wireless Communications and Mobile Computing, an associate editor of Wiley Security and Communication Networks, and an editorial board member of Journal of Networks, International Journal of E-Health and Medical Communications and KSII Transactions on Internet and Information Systems.He has served a technical program committee (TPC) co-chair for the IEEE WiMob'11 and $Q B S C^{\prime} 10$. He has served as a co-chair for various technical symposia of many international conferences, including the IEEE GLOBECOM and ICC. He has served as the TPC member for many international conferences, including the IEEE ICC, GLOBECOM, and WCNC. Dr. Li is a registered Professional Engineer (P.Eng.) in Canada and is a Senior Member of the IEEE and a member of the IEEE Communication Society, Computer Society, Vehicular Technology Society, and Ocean Engineering Society. 